\title{
DESIGN AND FABRICATION OF TWINROTOR UAV
}

\author{
Shlok Agarwal ${ }^{1}$, Apoorva Mohan ${ }^{2}$ and Kamlesh Kumar ${ }^{3}$ \\ ${ }^{1}$ Department of Mechatronics, Manipal University, India \\ shlokehotmail.co.in \\ ${ }^{2}$ Department of Mechatronics, Manipal University, India \\ priyam.apoorvaegmail.com \\ ${ }^{3}$ Department of Aeronautical and Automobile, Manipal University, India \\ kamlesh208755eaero.iisc.ernet.com
}

\begin{abstract}
There have been many advancements in the field of aerospace and avionics. Scientists have increasingly started to focus on VTOL (vertical take - off and landing) aircrafts. We have built a miniature VTOL twinrotor UAV. UAVs have begun to grab a lot of attention these days due to its numerous applications such as surveillance and relief. Twinrotor is a kind of a helicopter having two main propellers instead of one and no tail fin. All three important motion of the aircraft i.e. roll, pitch, yaw are controlled by thrust vectoring using servo motors and changing the magnitude of thrust using electronics speed controllers. The paper deals with the design of a basic UAV based on application and the construction keeping in mind the different concepts that govern its motion.
\end{abstract}

\section{KEYWORDS}

VTOL, UAV, Twinrotor

\section{INTRODUCTION}

\subsection{Unmanned Aerial Vehicles}

Unmanned Aerial Vehicles (UAV) are unmanned flying aircrafts. They are different from the commercial aircrafts and jets in a way that it does not have an on board pilot. Generally the pilot in a UAV controls the motion from the ground through a remote. Advanced development in the field has resulted in autonomous UAVs and the need of a pilot is eliminated. Such UAVs have an on-board controller that takes care of the stability and the trajectory motion of the UAV. Applications are often focused on the military areas, surveillance, inspection of transmission lines and power distribution; low cost filming and panoramic picturing for the movie industry, sport events, crop and herd monitoring, among others.

Over the past years many groups have worked on the development of UAV. The book Unmanned Aerial Vehicles by Randal and Beard [7] and Rogelio Lozana [8] give a very extensive explanation about the different types of UAVs and its modelling. The work done on quad rotors [1 2] gives a good idea on the design and stability of the four rotor system. [3 4] give a good idea about the control system and model of a quad rotor UAV. The model of a twinrotor can be related to this work. 
A smaller version of the UAV is termed as a Micro Air Vehicle (MAV). A MAV is a class of unmanned aerial vehicles (UAV) that has a size restriction and may be autonomous. Modern craft can be as small as 15 centimetres. Development is driven by commercial, research, government, and military purposes; with insect-sized aircraft reportedly expected in the future. The small craft allows remote observation of hazardous environments inaccessible to ground vehicles. A MAV weights much less than a UAV. A major advantage of a MAV over the UAV is that it consumes less power to do a similar application due to its less weight. A MAV also has better response time than a UAV because a smaller model would be more agile. [5 6] give a good description about the research and development in MAVs.

Another category of UAVs are fixed winged UAVS .These are popular when it comes to large size and long distance applications primarily used by the military. Fixed wing UAVs have enhanced payload carrying and delivering capabilities. Fixed wing UAVs are usually remote controlled and majority of them do not support vertical take-off and landing (VTOL).

\subsection{Determining a UAV based on Application}

Choosing a UAV depends upon the application it is being used for:

1) Fixed winged UAVS are used where large forward speed and long distance applications are required.

2) For steady surveillance or small applications the quad-rotor type UAV should be used.

3) When the UAV has critical applications in which it carries costly sensors or an application where fail-safe is required, then a redundant system is required hence hexrotor or oct-rotor is used.

4) Usually twin rotors are used for medium distance applications and low power consumption applications.

5) Much research is going on the tailsitter UAV or the single rotor UAV. It is very useful for low power application. The UAV supports VTOL and also fixed wing mode when it reaches a particular altitude.

\subsection{Twinrotor UAV}

A twin rotor-type UAV is a type of helicopter which is propelled by two rotors. The blades rotate in opposite directions and a tail rotor is not required in order to counter act the angular momentum of the propellers. As a coupled dynamical system, by altering the motor speed, the position is also changed. The system is under actuated and very dynamically unstable. In many situations it is desirable that the system is to be as small as possible to achieve large movements, being able to move both vertically and horizontally. Specific characteristics, such as vertical flight ability and flying at low speeds, allow the model to perform tasks which are difficult to implement through other mechanisms and structures. With demand of applications for this kind of aerial vehicle rapidly increasing, also increases the interest in research, both in industry and academics. Several studies are being conducted on the dynamics and describing methods to regulate their flight by adding automatic stability control through a diversity of hardware and software control schemes.

The objective of this work is to describe the design and the construction of the structure of the twinrotor device that can carry extra payloads. It is controlled by a remote transmitter that sends 
commands via radio to a microcontroller present on the twinrotor. This microcontroller is responsible for sending values of the speed for each rotor.

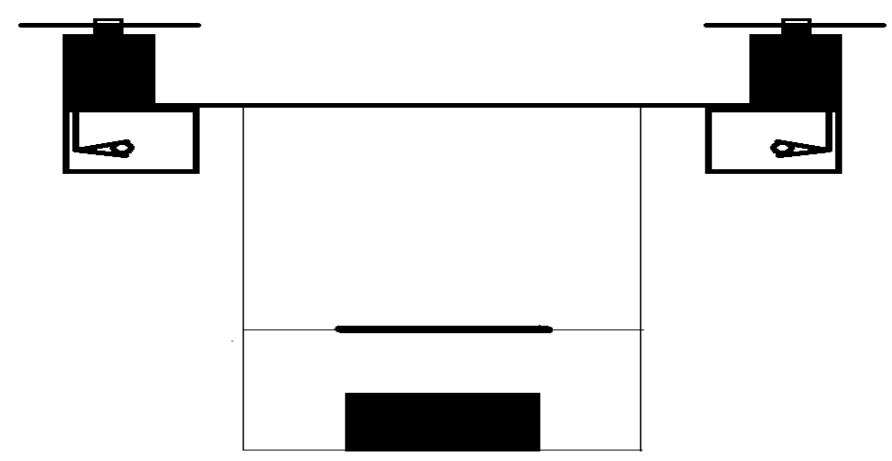

Figure 1.Skeleton Sketch

\section{Conceptual Design}

There is no particular design for a type of UAV. With new developing research, new designs are proposed. The reason for the on-going research in new designs is the unstable nature of the conventional designs. Every new design is aimed to be more stable and sophisticated than the previous one. A good design is the one which is more stable and manoeuvrable. Lower stability of design leads to complexity in designing the control system. The stable flight of a UAV heavily depends on the design. The motion of a UAV depends on the resultant forces and moments about the centre of gravity. The Newton-Euler Model gives us a good quantitative relation of the force and torque about the centre of gravity of a rigid body. For example, if a UAV needs to hover at a particular height, the moments about the centre of gravity need to be zero. The forces and moments applied at the centre of gravity depend on the structure and the design.

\subsection{Structure}

We have proposed a basic and an advanced structure. The advanced structure can only be implemented once stability is obtained in the basic structure. The advanced structure is more agile and can carry greater payloads.

\subsubsection{Initial Structure}

The structure we propose consists of two brushless DC motors (BLDC), two servo motors, controller board, battery, two ball bearings, a hollow aluminum U channel, an aluminum shaft, two sand boxes, and a light rectangular frame. The two ball bearings are coaxial to each other through a common aluminum shaft which runs right under the hollow aluminum channel. The sandboxes are fixed at the two ends of the channel with the ball bearings. The aluminum channel is rigidly attached to the main-frame which is a simple minimalistic rectangle having two shelves one for the controller board and one for the battery. The motors are arranged with parallel axis of rotation and rest on the two ends of the two sandboxes. The two servo motors are fixed at the ends of the aluminum channel. The servo arm is connected to the sand box with a $\mathrm{Z}$ link. The rectangular box was constructed using balsa planks.

In figure 1, the shaded part on the bottom shelf is the battery and the shaded part on middle shelf is the board. Motors and the propellers are mounted on the frame. 


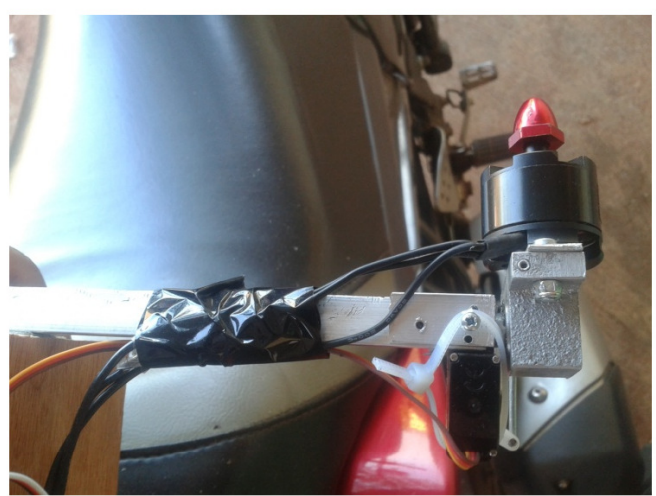

Figure 2(a)

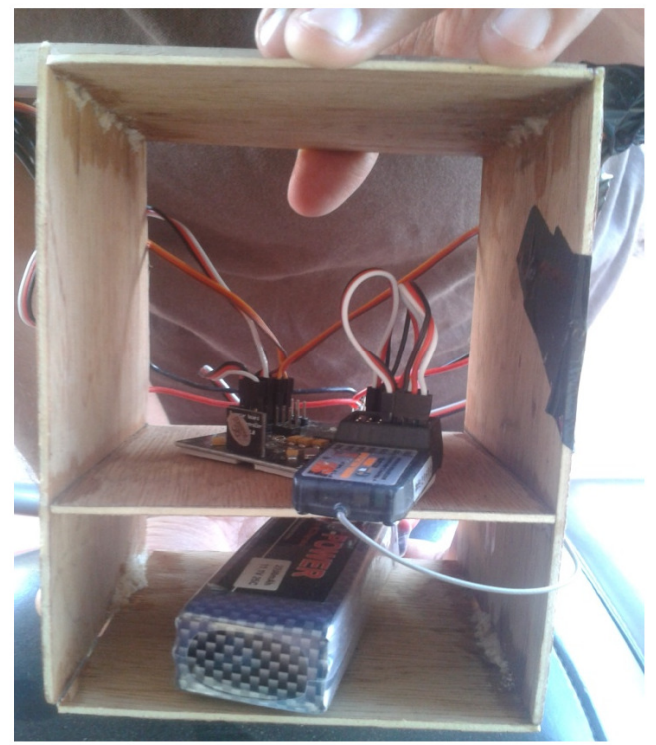

2(c)

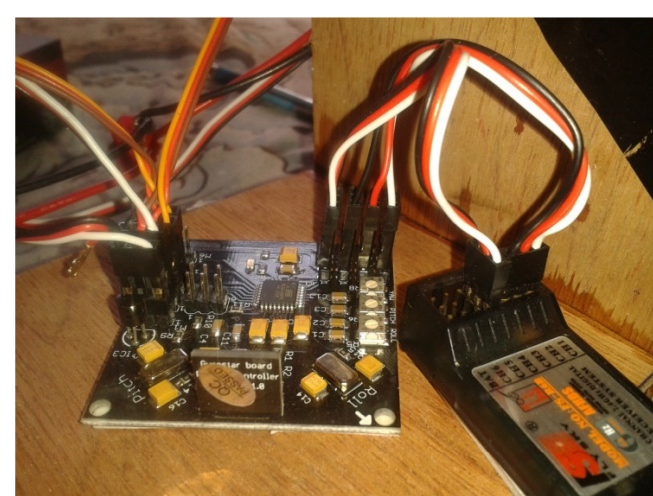

2(b)

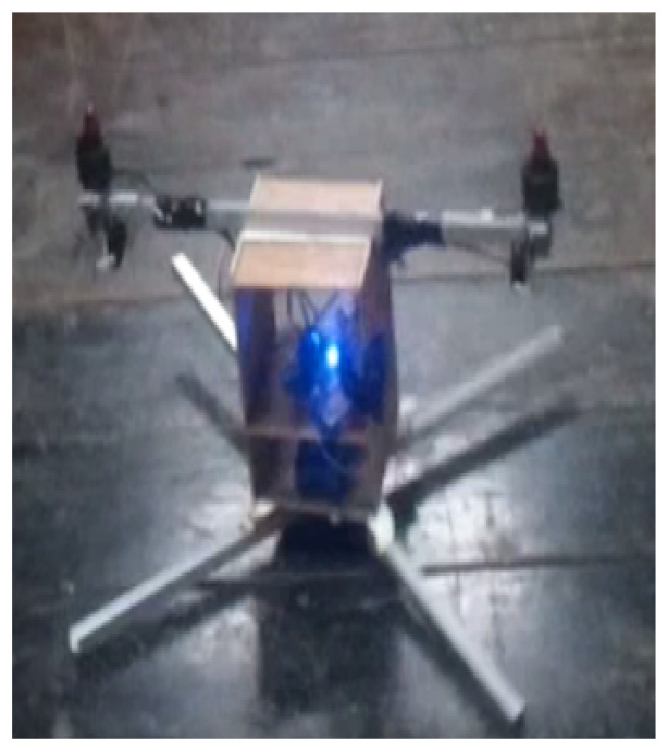

2(d)

Figure 2. Image shown at: (a) BLDC and Z joint, (b) Receiver and Controller board, (c) Shelf arrangement, (d) Complete assembled model

In any normal aircraft there is a propeller at the tail end to keep the copter in balance but in the proposed design there is no need of a tail fin to keep the aircraft in balance as the two propellers are counter rotatory which will keep the aircraft in balance.

As we can see the shape is kept rectangular to simplify the Center of Gravity (C.G) considerations. All the parts such as the board and the battery are placed such that their weight is distributed equally about the center of gravity. The battery was placed on the lower shelf so as to lower the C.G hence increasing stability.

The motors are placed equidistant from the center on opposite sides. The distance between the motors is such that there is no aerodynamic interaction between the propeller blades.

\subsubsection{Advanced structure}

The main structure consisting of balsa planks and aluminum stand are removed to reduce the weight and increase the payload. Due this there were fresh problems related to the placement of 
battery and the board. The board's orientation determines the stability of the copter so it had to be perfectly level placed. Hence we rigidly attached the board and battery at the center the aluminum channel that is attached to the motors. We also used carbon fiber rods in place of aluminum shaft to reduce the weight. We used thermocol pieces as supports. The battery was placed on the lower half so as to lower the C.G hence increasing stability. The motors are placed equidistant from the center on opposite sides. The distance between the motors is roughly adjusted such that there is no aerodynamic interaction between the propeller blades.

\subsection{Number of Rotors}

Unmanned aerial vehicles (UAVS) usually have three, four, six or even eight rotors for a stable flight. Motors are arranged in pairs placed radially opposite to each order to cancel out the torque in that direction. When there are 6 or 8 rotors the flight is more stable than in the case with 4 or 2 rotors. In case of 3 rotors, the 3 rotors form a plane in the orientation so this arrangement also obtains stable flight. With 4 or less motors a multi copter has the fly characteristics of a brick, and needs all the motors to overcome gravity. Once we go to 6 or more you start to introduce a level of redundancy in terms of ability to survive a motor out and still be able to crash safely. The drawback with these designs is the limited flight time. Using of two rotors provides enhanced flight time.

\subsection{Twinrotor Dynamics}

\subsubsection{Thrust Vectoring}

Thrust vectoring, also thrust vector control or TVC, is the ability of an aircraft, rocket, or other vehicle to manipulate the direction of the thrust from its engine(s) or motor in order to control the attitude or angular velocity of the vehicle.

For aircraft, the method was originally used to provide upward vertical thrust as a means to give aircraft vertical (VTOL) or short (STOL) takeoff and landing ability. Subsequently, it was realized that using vectored thrust in combat situations enabled aircraft to perform various maneuvers not available to conventional engine planes. To perform turns, aircraft that use no thrust vectoring must rely on aerodynamic control surfaces only, such as ailerons or elevator. Aircrafts with vectoring use control surfaces, but to a lesser extent.

In our model thrust vectoring is used to govern all the motions of the aircraft. This has been achieved by using servo motors that change the inclination of the motors thereby changing the direction of applied thrust. Various combinations of these changes produce different kinds of motion namely pitch yaw and roll. Thrust has been conveniently used to provide the copter its vertical takeoff and landing capabilities.

\subsubsection{Pitch, Roll and Yaw}

The twinrotor type UAV is driven by two BLDCs to produce thrust and two servo motors to give direction to the thrust. The rotors are rotated in opposite direction and placed radially opposite to each other in order to balance the torque. In Figure 3 we illustrate the direction of movement of the propellers with black arrows (the width is proportional to the speed of the rotors). Red arrows indicate the direction of the movement of the structure as whole. An increase (decrease) in the speed of the rotor causes the copter to move up (down) vertically. The variation of the two rotors placed on the same axis produces a torque around the axis generating an angular acceleration. Servo motors are used to tilt the motors to provide thrust vectoring, through which the forward and backward motion of the copter can be controlled. The three fundamental motions roll pitch and yaw movements are obtained as follows: 
Pitching is done by increasing the speed of rotation of the motors and inclining their axis of rotations in the same direction. This change in direction is brought by using servos.

Rolling is achieved by varying the speed of rotation in of either motor. The motor which has greater speed produces a higher thrust thus creating a rolling effect in opposite direction. Yawing is achieved by inclining the axis of rotation of BLDC opposite to each other. This is also done by using servos.

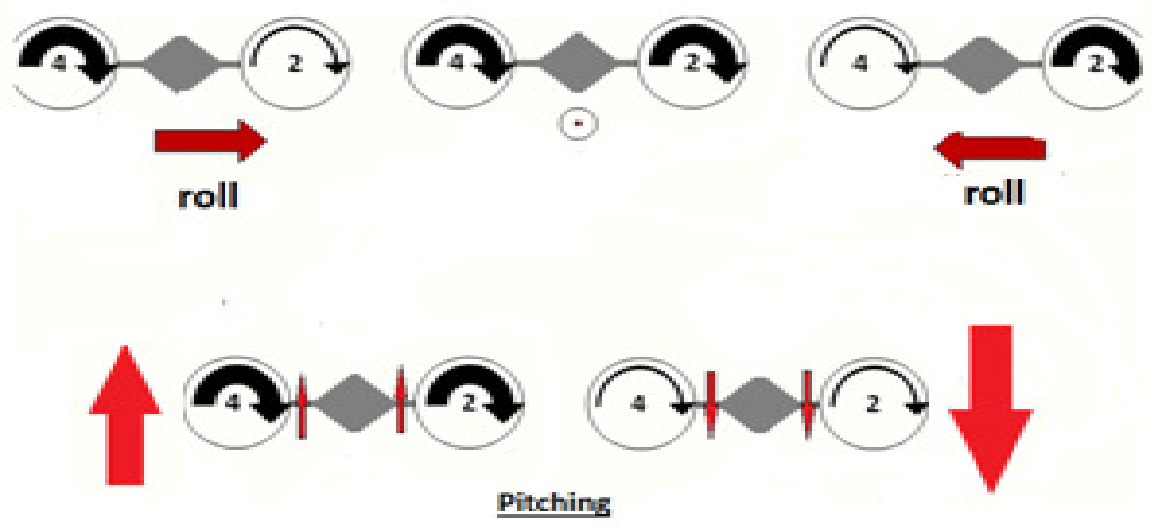

Figure 3. Demonstration of Roll and Pitch

\section{INSTRUMENTATION}

Along with a stable structure comes the need for sturdy instruments. The selection of instruments may vary with different applications. It is very important to understand how each instrument on the copter is interdependent with each other. Synchronization among reasonably compatible instruments is required for a stable flight.

\subsection{Motor and Propeller Selection}

Selection of BLDC depends on a lot of parameter. For such a model, motor requirements would be high KV (rpm/volts) ratings and high thrust. The motor should have less weight and have high efficiency. The rating of the motors depends on the specification of the propeller blade and payload required. The thrust produced depends on the diameter and pitch of the propeller. The servo motor should have flexible 180 degrees rotation and fast response.

The table below gives a approximate weight of the individual components of the twinrotor. The selection of BLDC, servo motors, electronic speed controllers and the battery vastly depend on the payload. For example, if the payload is high, a motor would need to have higher ratings. A higher rating motor would have more weight and size so higher rating servo motors would be required to move the BLDC. The electronic speed controller ratings depend on the ratings of the brushless DC Motors as higher rating BLDC would require higher current rating ESCs. Similarly higher rating components would require a high rating battery. Thus selections of instruments in a $\mathrm{UAV}$ are done as a set and not individually after thorough research. 
Table 1. Payload Calculations

\begin{tabular}{|c|c|c|}
\hline ITEM & QUANTITY & WEIGHT \\
\hline Brushless DC motor & 2 & $49 \times 2=80$ grams \\
\hline Servo motor & 2 & $40 \times 2=80$ grams \\
\hline Aluminium channel & 1 & 80 grams \\
\hline Aluminium shaft & 1 & 50 grams \\
\hline Frame[balsa wood] & 1 & 150 grams \\
\hline Control board & 1 & 30 grams \\
\hline Li-Po battery & 1 & 200 grams \\
\hline Miscellaneous & & 50 grams \\
\hline
\end{tabular}

Total approximate weight of the copter $=700$ grams

Thrust produced by one motor $=800$ grams

Thrust produced by both motors $=800 \times 2=1600$ grams

Working efficiency of the motor $=60 \%$

Payload capacity of the motors $=0.6 \times 1600=960$ grams

Thus, the UAV can bear an extra load of around 160 grams. The extra 160 grams could be used to carry a miniature surveillance camera. To increase the payload, the motors need to be changed and consequently an entire set of new BLDC, ESCs, Servos and battery would be required.

\subsection{Electronic Speed Control}

The flight control board firmware monitors measurement devices like gyros and accelerometers along with remote control inputs and sends signals to the ESCs attached to each motors. The ESCs use these inputs received from the flight control board to deliver the power required to control the speed of the motors. Each ESC has a processor, firmware and other electronics that manage this task by rapidly switching the power to the motor on and off. It has separate voltage regulator for the on board microcontroller.

\subsection{Communication}

The radios transmit data using digital signal at a frequency of $2.4 \mathrm{GHz}$. The motion of the copter is controlled with a 6 channel $2.4 \mathrm{GHz}$ transmitter \& receiver. It has a $0.8 \mathrm{~W}$ transmitter with range up to $1 \mathrm{~km}$ line of sight. The throttle to the motors is given by the left side of the remote. The servo motors and the varying speed of the BLDC are controlled by the spring based switches present on the right side of the remote control. The remote is fully programmable and it can be calibrated as per the convenience. The receiver is mounted on the copter and it works on a specified voltage of 4.8 volts.

\subsection{Battery and Power supply}

The lithium polymer battery is the most ideal battery for the applications which requires high current. It is used along with a protection circuit which is a microcontroller based smart circuit for protecting battery from deep discharge and becoming unstable if discharged below $9 \mathrm{~V}$. The battery consists of lithium polymerized at room temperature. If it is overcharged, it could lead to explosion of the battery. Observation suggests that improper use also leads to reduced current discharge from the battery. 


\subsection{Control Board}

The purpose of a control board is to stabilize the copter during flight. To do this, input from the gyroscopes (roll, pitch and yaw) are sent to the microcontroller. The three adjustable potentiometers increase or decrease gyro gain for all three axis (Roll, Pitch and Yaw) and can be used to calibrate the ESCs and reverse the gyro directions if necessary during pre-flight setup. The control board also uses signals from the radio system receiver $(\mathrm{Rx})$ and passes these signals to the microcontroller through the aileron, elevator, throttle and rudder inputs. The microcontroller then processes these signals according to the code burnt in it. It passes the control signals to the Electronic Speed Controllers (ESCs). These signals instruct the ESCs to make fine adjustments to the motors rotational speed which in turn stabilizes the Twin copter and helps control its motion.

\section{FLIGHT VALIDATION AND TESTING}

The structure was built bit by bit. The motors were first tested with different speeds and the thrust characteristics were obtained. It was noticed that for a particular speed maximum thrust was obtained. Any speed above it or below it reduced the thrust. This was tested using a spring arrangement where one end the spring was attached to the motor and the other was attached to the ground. The spring stiffness was known. Observing the deflection, the thrust applied by the motor could be predicted.

The remote needs to be calibrated so that for a given input, the motors get the same pulse at the starting for takeoff. Small mechanical inaccuracies in fabrication are taken care of by introducing trim settings in the remote unit. This gives permanent deflection (offset) to the servos to counteract for the inaccuracy.

For thrust vectoring, a $\mathrm{Z}$ joint was required between the servo arm and sandbox. The sandbox needs to be placed in perfect horizontal position while placing the cycle spoke ( $Z$ joint) in the servo arm.

\section{CONCLUSION}

In this paper we presented the mechanical structure and described all the parts comprising the development of a tiltrotor-type unmanned aerial vehicle. Such a design will be a good solution for a tiltrotor design when its dimension and cost are the main constrains. Thrust vectoring is clearly explained and illustrated. The principals involved are realized and proven accurately.An optimal motor and propellers orientation design is proposed to obtain the rolling, pitching and yawing movements. A few flight tests have been carried out to verify the finding.

We are working on the mathematical model and control system design of the twinrotor UAV at present and hope to get good results soon in the future.

\section{ACKNOWLEDGEMENTS}

The authors would like to thank Soumdutta Nath, Vedant Karia and Neil for their constant help and motivation during the project. The authors would also like to thank the Department of Mechatronics and Aeronautical at Manipal University for their support. 


\section{REFERENCES}

[1] Rejane Cavalcnte, André Luiz C. de Araújo, Design and Construction of a Quad rotor-type Unmanned Aerial Vehicle: Preliminary Results

[2] Swee King Phang, Chenxiao Cai, Ben M. Chen, Tong Heng Lee, Design and Mathematical Modeling of a 4-Standard-Propeller(4SP) Quadrotor

[3] N. Kundak and B. Mettler, "Experimental Framework for Evaluating Autonomous Guidance and Control Algorithms for Agile Aerial Vehicles," European Control Conference,2007, pp. 293-300.

[4] A. Tayebi, and S. McGilvray. Attitude Stabilization of a VTOL Quadrotor Aircraft, IEEE Transactions on Control Systems Technology, Vol. 14, pp. 562-571, 2006

[5] Morris, S.Design and flight test results for micro-sizedfixed-wing and VTOL aircraft. In Proceedings of The First International Conference on Emerging Technologies for Micro Air Vehicles, Georgia Institute of Technology, Atlanta, GA, 1997, pp. 1-14.

[6] Watkins, S.Development of a micro air vehicle. The Aeronaut. J., 2003,107, 117-123.

[7] Randal and Beard, Unmanned Aerial Vehicles

[8] Rogelio Lozana, Unmanned Aerial Vehicles

[9] Diydrones. http://www.diydrones.com/

[10] RCdhamaka http://www.rcdhamaka.com/

\section{Authors}

Shlok Agarwal is a final year BE student at Manipal Institute of Technology in the Department of Mechatronics. His current research interests include robotics, automation embedded systems and control system design.

Apoorva Mohan is a final year BE student at Manipal Institute of Technology in the Department of Mechatronics. His current research interests include mechanical system design and autotronics.

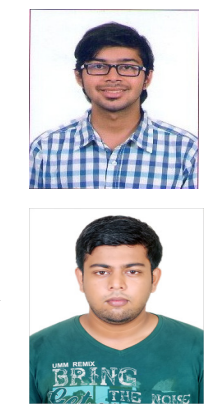

Kamlesh Kumar is an Assistant professor at Manipal Institute of Technology in the Department of Aeronautical and Automobile. He has completed his masters in aerospace from Indian institute of Science, Bangalore. His current research interests include Navigation, Guidance and Control, Aviation Electronics and Flight Dynamics

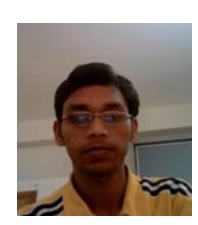

